\title{
Small Dense LDL: New Marker for Cardiovascular Risk Assessment and its Therapeutic Inflection
}

M Salman Khan*

Department of Biotechnology, Integral University, Lucknow-226026, India

Keywords: Low density lipoprotein; Small dense LDL; Cardiovascular disease

Abbreviations: Cardiovascular Disease (CVD); Coronary Heart Disease (CHD); Low Density Lipoprotein (LDL); Small Dense LDL (Sd-LDL)

Cardiovascular diseases (CVD) encompass any medical conditions related to the heart and blood vessels. CVD is the main cause of disability and premature death worldwide, and is projected to remain the leading cause of death. An estimated 17.5 million people died from this disease in 2005, representing 30\% of all global deaths. Of these deaths, 7.6 million were due to coronary heart disease (CHD) and 5.7 million because of stroke. If immediate and proper attention is not paid, by 2015 an estimated 20 million people will die from CVD, including stroke [1]. In terms of attributable deaths, the leading cardiovascular risk factor globally, is raised blood pressure (to which $13 \%$ of global deaths is attributed), followed by tobacco use (9\%), raised blood glucose (6\%), physical inactivity (6\%) and overweight and obesity (5\%). CVD also accounts for about $60 \%$ of all mortality in people with diabetes.

Persons with high levels of cholesterol in blood, a condition called as hypercholesterolemia, are more prone to atherosclerosis. It is not a disease but a metabolic derangement that can be caused by many diseases, notably cardiovascular disease. "Hyperlipidemia" (elevated levels of lipids in the blood) and "hyperlipoproteinemia" (elevated levels of lipoproteins in the blood) are the other factors responsible for CVD. Elevated cholesterol in the blood is due to abnormalities in the levels of lipoproteins, the particles that carry cholesterol in the bloodstream.

Cholesterol contains various subtypes that include very-lowdensity lipoprotein cholesterol (VLDL-C), low density lipoprotein cholesterol (LDL-C) and high density lipoprotein cholesterol (HDL-C). The normal cholesterol range in human body should be below $200 \mathrm{mg} /$ $\mathrm{dl}$ and is good for body to function normally and anything over $240 \mathrm{mg} /$ $\mathrm{dl}$ indicates risk for developing CVD. Relative risk of all lipoproteins in CVD has been extensively studied and till now LDL-C, known as bad cholesterol, is considered as a marker for cardiovascular risk assessment [2]. The LDL-C level in human body should be below $130 \mathrm{mg} / \mathrm{dl}$. High density lipoprotein cholesterol, which is known as good cholesterol and have antioxidant property, should range between $35-40 \mathrm{mg} / \mathrm{dL}$. The high level of HDL-C $(\sim 60 \mathrm{mg} / \mathrm{dL})$ has the benefit of reducing the chances of heart attack. Moreover, there are several amendable risk factors that include hyperlipidemia, dyslipidemia and have higher incidence of deranged lipid profile. The various study established that elevated LDL-C and increased ratio of LDL/HDL (high density lipoprotein) is the most important lipid derangement making those subjects more susceptible to atherosclerosis [3].

\section{Small dense LDL: A New Marker}

Modern research on CVD decode several new risk factors that include elevated lipoprotein(a), high sensitivity $\mathrm{C}$ reactive protein (hsCRP), fibrinogen and hyper-homocysteinemia etc., an important one of which is estimation of small dense LDL cholesterol (sd LDL-C).
Low density lipoproteins are composed of distinct subclasses that differ in size, density, chemical composition, and their association with cardiovascular disease. Numerous studies have clearly demonstrated the various sub-fractions of LDL by using different methods like density gradient, ultracentrifugation, PAGE, NMR etc [4-6] and seven distinct LDL subspecies were identified depending on their densities, which differ in their metabolic behavior and pathological roles $[7,8]$. Two phenotypes of LDL were identified-pattern A with LDL $>25.5 \mathrm{~nm}$ (large and buoyant) and pattern B with LDL $\leq 25.5 \mathrm{~nm}$ (small and dense)

Lipoprotein profiles that are relatively rich in sd-LDL particles are associated with up to a 3-fold greater risk of myocardial infarction than those mainly consist of large buoyant (lb)-LDL particles. Currently published report established that predominance of sd-LDL-C is a major component of an atherogenic lipoprotein phenotype, and a source of increased risk for coronary heart disease. The ratio of sd-LDL-C to LDL-C plays an important role in assessing CVD. Researches established that sd-LDL cholesterol and lipoprotein level in normolipidemic condition is $\sim 30 \%$ of total LDL-C in blood. In hyperlipidemic animal and human subject this ratio is increased by several folds depending upon the degree and severity of disease [911]. It has been shown that not only the prevalence of sd-LDL but its concentration was substantially increased in healthy subjects with various types of hyperlipidemia such as hypercholesterolemia(LDL), hypertriglyceridemia, combined hyperlipidemia and chylomicronemia, hyperlipidimea with $\mathrm{CHD}$, type 2 diabetic patients with and without CHD [9]. Similarly, Quebec cardiovascular study has confirmed that a predominance of sd-LDL is a strong and independent predictor of CHD in the first seven years of follow-up [12]. Another report specifies that progression of CHD was closely linked not to the LDL particle size, but to the concentration of sd-LDL [13].

\section{Atherogenic Properties of Oxidized LDL and Small Dense LDL}

Evidence suggests that common risk factors for atherosclerosis increase the risk of the production of reactive oxygen species (ROS), not only from the endothelial cells, but also from the smooth muscle cells and the adventitial cells. That include: hypercholesterolemia or dyslipoproteinemia, diabetes mellitus (DM), arterial hypertension, smoking, age etc. According to the theory of oxidative stress,

*Corresponding author: M.Salman Khan, Department of Biotechnology, Integra University, Lucknow-226026, India, E-mail: contactskhan@gmail.com

Received September 14, 2012; Accepted September 14, 2012; Published September 14, 2012

Citation: Khan MS (2012) Small Dense LDL: New Marker for Cardiovascula Risk Assessment and its Therapeutic Inflection. Biochem Anal Biochem 1:e118. doi:10.4172/2161-1009.1000e118

Copyright: (C) 2012 Khan MS. This is an open-access article distributed under the terms of the Creative Commons Attribution License, which permits unrestricted use, distribution, and reproduction in any medium, provided the original author and source are credited. 
atherosclerosis is the result of the oxidative modification of LDL in the arterial wall by ROS.

Oxidative modification of lipoproteins is believed to play a central role in the pathogenesis of atherosclerosis [14]. Because plasma contains several antioxidants and lipoproteins with oxidative damage have been isolated from atherosclerotic lesions, lipoprotein oxidation generally is considered to occur in the vessel wall [14]. Research has shown that human atherosclerotic plaques contain massive amounts of lipid peroxidation products, despite the presence of large quantities of a-tocopherol (vitamin E) and ascorbate Suarna et al. [15]. Therefore, it is unclear whether oxidized lipoproteins originate in the arterial wall or are produced in the circulation and then enter the intimal space. Although LDL is a major risk factor for CAD, experiments have shown that incubation of macrophages with native LDL does not result in foam cell formation, a characteristic feature of atheromatous lesions [16]. However, after chemical modification of LDL, rapid uptake occurs, leading to cholesterol accumulation in the macrophage with subsequent foam cell formation, which suggests that LDL modification is a necessary step in atherogenesis. Oxidized (OX) LDL can induce foam cell formation, and oxidative modification of LDL is now recognized as an important process that occurs in vivo Steinberg [14]. Several lines of research support the occurrence of LDL oxidation in vivo [14].

Many cell types are capable of oxidizing LDL, including monocytes, macrophages, neutrophils, endothelial cells, smooth muscle cells and fibroblasts. However, cell types that are involved in the atherosclerotic lesion in which OX LDL is found, i.e. macrophages, endothelial cells and smooth muscle cells, would seem to be the most likely to contribute to LDL oxidation in vivo. Once initiated, oxidation of LDL is a free radical driven lipid peroxidation chain reaction. Lipid peroxidation is initiated by free radical attack on double bond associated with a polyunsaturated fatty acid (PUFA). This results in the removal of a hydrogen atom from a methylene $\left(\mathrm{CH}_{2}\right)$ group, the rate of which determines the rate of initiation, a key step in lipid peroxidation. Molecular rearrangement of the resulting unstable carbon radical results in a more stable configuration, a conjugated diene. The conjugated diene reacts very quickly with molecular oxygen, and the peroxyl radical thus formed is a crucial intermediate. A PUFA peroxyl radical in LDL may abstract a hydrogen atom from an adjacent PUFA to form a hydroperoxide and another lipid radical, a reaction which results in chain propagation. Removal of hydrogen atoms by the peroxyl radical from other lipids, including cholesterol, eventually yields oxysterols. Lipid hydroperoxides fragment to shorter-chain aldehydes, including malondialdehyde and 4-hydroxynonenal. These reactive aldehydes in turn may bind to $\varepsilon$-amino groups of apo B-100, giving the protein an increased net negative charge. The classical LDL receptor recognizes a specific domain of positive charges from lysine, arginine and histidine residues on apo B. Alteration of this domain results in failure of binding by the apo $\mathrm{B} / \mathrm{E}$ receptor and an increase in negative surface charge on apo B-100 results in increased recognition by the scavenger receptor. In vitro LDL oxidation have verified the existence of "lag phase", during which significant oxidation of LDL cannot be detected, prior to the onset of the "propagation phase", presumably after the endogenous antioxidants have been consumed. A steady increase in the detectable byproducts of oxidation continues, until the substrate, i.e. the PUFAs, has been depleted, and a plateau phase is reached. Assessment of resistance of LDL to oxidation has generally involved in measurement of the duration of the lag phase including other variables, such as the rate of propagation, are also noted.
The increased atherogenecity of LDL is associated with a preponderance of small dense (sd-) LDL subpopulation that is more prone to oxidative modification than large buoyant (lb-) LDL [17]. Lipoprotein profiles that are relatively rich in sd-LDL particles are associated with up to a 3-fold greater risk of myocardial infarction than those mainly consist of lb-LDL particles $[18,19]$. However, the mass of sd-LDL is independently associated with disease risk $[19,20]$, suggesting that these particles are more directly atherogenic than lbLDL. It has been suggested that in comparison to lb-LDL or LDL, sdLDL are highly atherogenic as a result of their enhanced susceptibility to oxidative modification, higher penetration in the arterial wall, their lower binding affinity for the LDL-receptor, and prolonged plasma half life [21]. Differences in oxidative susceptibility between lb-LDL and sd-LDL have been attributed to differences in their physicochemical properties. Relative to $\mathrm{lb}$-LDL, sd-LDL have a reduced content of antioxidants and free cholesterol [22-25], and are enriched with polyunsaturated fatty acids [22] and possibly, hydroperoxides [26]. Antioxidant concentrations and free cholesterol content have been shown to predict differences in the oxidative susceptibility of LDL density subfraction $[21,22,25]$. Therefore, in view of above evidence, sdLDL has now been highlighted as a new powerful and useful marker for the risk of hyperlipidimea with and without CVD/CHD, type 2 diabetic patients with and without CVD/CHD.

\section{Therapeutic approaches: Role of Natural Products}

Conventional treatment of atherogenic lipid profile aims at reducing total cholesterol levels and LDL cholesterol or increasing HDL cholesterol by using different treatment available. The most important and widely used drugs for the treatment of hypercholesterolemia are Statins. They reduce the cholesterol level by inhibiting HMG-CoA reductase enzymatic activity, the rate limiting enzyme in biosynthetic pathway of cholesterol. These drugs decrease intracellular cholesterol, increase LDL receptors and accelerate removal of LDL-C and TGs-rich lipoprotein and constitute the first line of treatment for reducing LDL-C. Though statins are superior to all other medications for reducing LDL-C concentration but their effect on sd LDL-C is variable $[27,28]$. Both fluvastatin and atorvastatin have been shown to shift the LDL profile towards more buoyant particles but pravastatin and simvastatin have not shown any such result. In addition, in hyperlipidemic patients with type 2 diabetes, low doses of pitavastatin $(1 \mathrm{mg})$ and fenofibrate (100 $\mathrm{mg}$ ) were both effective in decreasing sd-LDL-C concentration but via a different mechanisms: the former decreases total LDL including sdLDL, while the latter decreased sd-LDL specifically [29]. Furthermore, in diabetic patients with mixed dyslipidemia, combination therapy with simvastatin and fenofibrate had a greater positive effect on LDL cholesterol size profile, with a shift from more atherogenic sd-LDL-C to less atherogenic $\mathrm{lb}-\mathrm{LDL}-\mathrm{C}$, than either fenofibrate or simvastatin monotherapy [30].

Although statins are relatively safe and well tolerated, there are still significant numbers of patients who cannot tolerate them because of their high costs, especially in India, and lots of side effects, such as, Liver Enzyme Elevation (AST, ALT), muscle pain and weakness, headaches or fevers, loss of memory and brain function, stomach pain, weight loss, vomiting etc. In order to exonerate these side effects and to develop new drugs that are not only capable of reducing cholesterol level but also inhibits LDL and sd-LDL oxidation, existing research are now focused on natural products which have no side effects, cost effective and easily available. Search of new agents that have both lipid lowering as well as antioxidant property are going on. 
There are some natural antioxidant enzymes as well as low molecular weight antioxidants, which are subdivided into lipid-soluble antioxidants (tocopherol, carotenoids, quinones, bilirubin and some polyphenols) and water soluble antioxidants (ascorbic acid, uric acid and polyphenols). These delay or inhibit cellular damage mainly through free radical scavenging property. Apart from this there are some natural compounds also which may be used as a combined source for treating oxidative stress and atherosclerosis such as, Vitamin A, C, E, K, quercitin, curcumin, retinol, catechin, thymoquinone, lutin, lycopene etc. Preclinical and clinical studies showed that some of these compounds possess antiatherogenic activity but detailed investigation regarding their mechanism of action is not done, except for Vitamin E that contains tocotrienols and tocopherols. Tocotrienols are now considered to be more potent antioxidant and hypolipidemic agent than tocopherol $[10,11]$. Like statins, tocotrienols also works by suppressing HMG-CoA reductase enzymatic activity.

The disappointing results of antioxidant strategies, in both the prevention and limitation of atherogenesis and cardiovascular complications in humans, raise questions about the role of oxidative modification of LDL in atheromatosis. Numerous reasons could explain the failure of antioxidants in the prevention and treatment of cardiovascular diseases. There are points that need further examination, such as the fact that random generation of free radicals cannot explain the progression rate of atherosclerosis, the level of oxidative modification of LDL, in what way the oxidative process contributes to the reaction of atherosclerotic plaque and why atherosclerotic lesions occur at specific sites on vessel wall, which antioxidants best inhibit endothelial dysfunction, proliferation of smooth muscle cells, hypertrophy and lipid oxidation, which patient groups respond better to antioxidant treatment, and what are the required doses of antioxidants and in what way may the side-effects, such as DNA damage after the high dose administration of vitamin $\mathrm{C}$ be confronted.

From the above discussion it is clear that sd-LDL is now recognized marker and therapeutic target for CVD risk assessment and management. Moreover, there is a large body of evidence connecting the effects of oxidative stress with atherogenesis. However, so far we have not determined a specific causative relation between oxidative events in general and oxidative modification of LDL and sd-LDL in particular with respect to atherosclerosis. Further, with the contribution of molecular cardiology and pharmacogenetics, to elucidate the molecules as well as the inhibiting mechanisms that interfere in the oxidative process is needed. Thus, the therapeutic inflection of sdLDL often require the combination of different treatment of drugs, lifestyle modification and dietary intake, which may constitute both primary and secondary prevention of CVD. In addition, individualized treatment is required for proper assessment of various risk factors that promotes CVD, particularly sd-LDL. In last, the lipid lowering agent whether they are natural or chemically synthesized may constitute the first line of therapy along with life style modification.

\section{References}

1. WHO (2005) Preventing chronic disease: a vital investment. Geneva.

2. National Cholesterol Education Program (NCEP) (2001) Executive summary of the third report of the NCEP expert panel on detection evaluation treatment of high blood cholesterol in adults (Adult Treatment Panel III). J Am Med Assoc 285: 2486-2497.

3. Sharma SB, Dwivedi S, Prabhu KM, Singh G, Kumar N, et al. (2005) Coronary risk variables in young asymptomatic smokers. Indian J Med Res 122: 205-210.

4. Swinkels DW, Hak-Lemmers HL, Demacker PN (1987) Single spin density gradient ultracentrifugation method for the detection and isolation of light and heavy low density lipoprotein subfractions. J Lipid Res 28: 1233-1239.

5. Krauss RM, Blanche PJ (1992) Detection and quantification of LDL subfractions Curr Opin Lipidol 3: 377-383.

6. Otvos JD, Jeyarajah EJ, Bennett DW, Krauss RM (1992) Development of proton nuclear magnetic resonance spectroscopic method for determining plasma lipoprotein concentrations and subspecies distributions from a single, rapid measurement. Clin Chem 38: 1632-1638.

7. Berneis KK, Krauss RM (2002) Metabolic origins and clinical significance of LDL heterogeneity. J Lipid Res 43: 1363-1379.

8. Campos H, Blijlevens E, McNamara JR, Ordovas JM, Posner BM, et al. (1992) Low density lipoprotein particle size distribution: results from the Framingham Offspring Study. Arterioscler Thromb 12: 1410-1419.

9. Hirano T, Ito Y, Koba S, Toyoda M, Ikejiri A, et al. (2004) Clinical significance of small dense low-density lipoprotein cholesterol levels determined by the simple precipitation method. Arterioscler Thromb Vasc Biol 24: 558-563.

10. Salman Khan M, Akhtar S, Al-Sagair OA, Arif JM (2011) Protective effect of dietary tocotrienols against infection and inflammation-induced hyperlipidemia: an in vivo and in silico study. Phytother Res 25: 1586-1595.

11. Khan MS, Khan MK, Siddiqui MH, Arif JM (2011) In vivo and In silico approach to elucidate the tocotrienols mediated fortification against infection and inflammation induced alterations in antioxidant defense system. Eur Rev Med Pharm Sci 15: 916-930.

12. St-Pierre AC, Cantin B, Dagenais GR, Mauriège P, Bernard PM, et al. (2005) Low density lipoprotein subfractions and the long-term risk of ischemic heart disease in men, 13 year follow-up data from the Quebec cardiovascular study. Arterioscle Thromb Vasc Biol 25: 553-559.

13. Koba S, Hirano T, Ito Y, Tsunoda F, Yokota Y, et al. (2006) Significance of small dense low-density lipoprotein-cholesterol concentrations in relation to the severity of coronary heart diseases. Athersclerosis 189: 206-214.

14. Steinberg D (1997) Lewis A. Corner Memorial Lecture. Oxidative modification of LDL and atherogenesis. Circulation 95: 1062-1071.

15. Suarna C, Dean RT, May J, Stocker R (1995) Human atherosclerotic plaque contains both oxidized lipids and relatively large amounts of a-tocopherol and ascorbate. Arterioscler Thromb Vasc Biol 15: 1616-1624.

16. Brown MS, Goldstein JL (1983) Lipoprotein metabolism in the macrophage: implications for cholesterol deposition in atherosclerosis. Ann Rev Biochem 52 223.

17. Chancharme L, Thérond P, Nigon F, Lepage S, Couturier M, et al. (1999) Cholesteryl ester hydroperoxide liabilities key feautrue of the oxidative susceptibility of small dense LDL. Arterioscler Thromb Vasc Biol 19: 810-820.

18. Austin MA, Hokanson JE, Brunzell JD (1994) Characterization of low-density lipoprotein subclasses: methodologic approaches and clinical relevance. Curr Opin Lipidol 5: 395-403.

19. Stampfer MJ, Krauss RM, Ma J, Blanche PJ, Holl LG, et al. (1996) A prospective study of triglyceride level, low-density lipoprotein particle diameter, and risk of myocardial infarction. J Am Med Assoc 276: 882-888.

20. Gardner CD, Fortmann SP, Krauss RM (1996) Association of small low-density lipoprotein particles with the incidence of coronary artery disease in men and women. J Am Med Assoc 276: 875-81.

21. Chapman MJ, Guerin M, Bruckert E (1998) Atherogenic, dense low-density lipoproteins: pathophysiology and new therapeutic approaches. Eur Heart $J$ 19: A24-A30.

22. de Graaf J, Hak-Lemmers HL, Hectors MP, Demacker PN, Hendriks JC, et al. (1991) Enhanced susceptibility to in vitro oxidation of the dense low-density lipoprotein subfraction in healthy subjects. Arterioscler Thromb 11: 298-306.

23. Tribble DL, Holl LG, Wood PD, Krauss RM (1992) Variations in oxidative susceptibility among six low-density lipoprotein subfractions of varying size and density. Atherosclerosis 93: 189-199.

24. Tribble DL, Rizzo M, Chait A, Lewis DM, Blanche PJ, et al. (2001) Enhanced oxidative susceptibility and reduced antioxidant content of metabolic precursor of small, dense low-density lipoproteins. Am J Med 110: 103-110.

25. Tribble DL, van den Berg JJ, Motchnik PA, Ames BN, Lewis DM, et al. (1994) 
Citation: Khan MS (2012) Small Dense LDL: New Marker for Cardiovascular Risk Assessment and its Therapeutic Inflection. Biochem Anal Biochem 1:e118. doi:10.4172/2161-1009.1000e118

Oxidative susceptibility of low-density lipoprotein subfractions is related to their ubiquinol-10 and alpha-tocopherol content. Proc Natl Acad Sci, USA. 94: 11831187.

26. Sevanian A, Hwang J, Hodis H, Cazzolato G, Avogaro P, et al. (1996) Contribution of an in vivo oxidized LDL to LDL oxidation and its association with dense LDL subpopulations. Arterioscler Thromb Vasc Biol 16: 784-793.

27. McKenney JM, McCormick LS, Schaefer EJ, Black DM, Watkins ML (2001) Effect of niacin and atorvastatin on lipoprotein subclasses in patients with atherogenic dyslipidemia. Am J Cardiol 88: 270-274.

28. Forster LF, Stewart G, Bedford D, Stewart JP, Rogers E , et al. (2002) Influence of atorvastatin and simvastatin on apolipoprotein $\mathrm{B}$ metabolism in moderate combined hyperlipidemic subjects with low VLDL and LDL fractional clearance rates. Atherosclerosis 164: 129-145.

29. Tokuno A, Hirano T, Hayashi T, Mori Y, Yamamoto T, et al. (2007) The effects of Statin and Fibrate on lowering small dense LDL-Cholesterol in hyperlipidemic patients with type 2 diabetes. J Ather Thromb 14: 128-132.

30. May HT, Anderson JL, Pearson RR, Jensen JR, Horne BD, et al. (2008) Comparison of Effects of Simvastatin Alone Versus Fenofibrate Alone Versus Simvastatin Plus Fenofibrate on Lipoprotein Subparticle Profiles in Diabetic Patients With Mixed Dyslipidemia (from the Diabetes and Combined Lipid Therapy Regimen Study). Am J Cardiol 101: 486-489. 